\title{
Prediction of fatty acids in chocolates with emphasis on C18:1 trans fatty acids positional isomers using ATR-FTIR associated with multivariate calibration
}

Tatiane Lima Amorim ${ }^{\mathrm{ab}}$, Lucas Mattos Duarte ${ }^{\mathrm{bc}}$, Marcone Augusto Leal de Oliveira ${ }^{\mathrm{b}^{*}}$, Miguel Angel de la Fuente ${ }^{a}$, Pilar Gómez-Cortés ${ }^{a}$,

a Instituto de Investigación en Ciencias de la Alimentación (CIAL, CSIC-UAM), Universidad Autónoma de Madrid, Nicolás Cabrera, 9, 28049, Madrid, Spain.

b Grupo de Química Analítica e Quimiometria (GQAQ), Departamento de Química, Universidade Federal de Juiz de Fora, José Lourenço Kelmer, 36036-900, Juiz de Fora, MG, Brazil.

c Departamento de Química Analítica, Instituto de Química, Universidade Federal Fluminense, 24020-141, Niterói, RJ, Brazil 
Table 1 Supporting Information. GC-FID and ATR-FTIR data considering calibration (21 samples) and prediction (11 samples) steps of multivariate calibration for fatty acids groups. Results are expressed as $\mathrm{g} / \mathbf{1 0 0} \mathrm{g}$ total fatty acids;

\begin{tabular}{|c|c|c|c|c|c|c|c|c|c|c|c|c|c|c|c|c|}
\hline & \multicolumn{4}{|c|}{$\Sigma$ TFA } & \multicolumn{4}{|c|}{$\Sigma$ SFA } & \multicolumn{4}{|c|}{$\Sigma$ MUFA } & \multicolumn{4}{|c|}{$\Sigma$ PUFA } \\
\hline & \multicolumn{2}{|c|}{ Calibration } & \multicolumn{2}{|c|}{ Prediction } & \multicolumn{2}{|c|}{ Calibration } & \multicolumn{2}{|c|}{ Prediction } & \multicolumn{2}{|c|}{ Calibration } & \multicolumn{2}{|c|}{ Prediction } & \multicolumn{2}{|c|}{ Calibration } & \multicolumn{2}{|c|}{ Prediction } \\
\hline & GC- & ATR- & GC- & ATR- & GC- & ATR- & GC- & ATR- & GC- & ATR- & GC- & ATR- & GC- & ATR- & GC- & ATR- \\
\hline & FID & FTIR & FID & FTIR & FID & FTIR & FID & FTIR & FID & FTIR & FID & FTIR & FID & FTIR & FID & FTIR \\
\hline \multirow{21}{*}{ 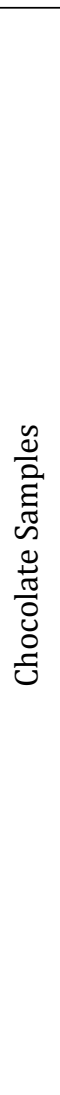 } & 2.15 & 1.94 & 3.87 & 3.65 & 84.81 & 86.17 & 78.52 & 80.52 & 13.59 & 13.11 & 19.39 & 15.57 & 0.73 & 0.99 & 0.92 & 1.86 \\
\hline & 2.38 & 2.32 & 0.35 & 0.54 & 93.2 & 94.84 & 97.16 & 96.99 & 5.74 & 8.38 & 2.23 & 6.74 & 0.58 & 0.74 & 0.47 & 0.20 \\
\hline & 0.55 & 0.63 & 0.41 & 0.41 & 61.31 & 61.02 & 61.14 & 60.87 & 31.13 & 31.36 & 33.04 & 32.68 & 4.96 & 4.76 & 3.44 & 3.52 \\
\hline & 0.38 & 0.90 & 7.9 & 7.57 & 60.19 & 59.01 & 19.03 & 18.88 & 31.16 & 32.64 & 39.12 & 39.19 & 5.88 & 4.91 & 32.69 & 33.27 \\
\hline & 0.36 & 0.51 & 0.35 & 0.40 & 62.51 & 61.72 & 63.22 & 64.19 & 32.17 & 33.84 & 30.55 & 31.77 & 3.06 & 3.30 & 3.79 & 2.59 \\
\hline & 7.53 & 6.82 & 0.46 & 0.42 & 19.55 & 19.48 & 63.93 & 63.58 & 39.31 & 38.42 & 31.08 & 30.99 & 32.11 & 31.78 & 2.68 & 2.79 \\
\hline & 0.39 & 0.44 & 0.3 & 0.28 & 63.98 & 63.97 & 50.89 & 53.24 & 31.12 & 33.23 & 40.87 & 34.70 & 2.74 & 2.83 & 4.93 & 5.98 \\
\hline & 0.40 & 0.4 & 0.23 & -0.08 & 63.95 & 63.8 & 61.00 & 61.59 & 31.24 & 32.1 & 33.58 & 29.43 & 2.75 & 2.70 & 2.99 & 3.09 \\
\hline & 0.35 & 0.46 & 0.71 & 0.9 & 63.93 & 63.73 & 62.61 & 63.3 & 31.02 & 29.79 & 31.84 & 31.92 & 2.76 & 3.06 & 2.99 & 3.19 \\
\hline & 0.39 & 0.29 & 0.57 & 0.48 & 64.04 & 64.6 & 59.96 & 61.26 & 30.8 & 29.9 & 33.49 & 31.13 & 2.86 & 3.02 & 4.01 & 4.21 \\
\hline & 0.46 & 0.36 & 0.81 & 0.94 & 63.94 & 64.38 & 61.11 & 60.88 & 31.05 & 30.72 & 32.94 & 33.45 & 2.7 & 2.63 & 3.38 & 3.48 \\
\hline & 0.31 & 0.56 & - & - & 96.01 & 92.89 & - & - & 3.21 & 8.20 & - & - & 0.49 & 0.17 & - & - \\
\hline & 0.72 & 0.68 & - & - & 62.82 & 63.4 & - & - & 32.14 & 31.15 & - & - & 2.7 & 2.99 & - & - \\
\hline & 0.56 & 0.54 & - & - & 63.8 & 62.96 & - & - & 31.25 & 33.63 & - & - & 2.64 & 2.61 & - & - \\
\hline & 0.65 & 0.74 & - & - & 62.68 & 62.32 & - & - & 31.81 & 31.3 & - & - & 2.97 & 2.8 & - & - \\
\hline & 0.71 & 0.69 & - & - & 62.62 & 63.42 & - & - & 31.99 & 32.71 & - & - & 2.87 & 2.84 & - & - \\
\hline & 0.57 & 0.38 & - & - & 59.47 & 60.04 & - & - & 31.97 & 32.36 & - & - & 5.82 & 5.68 & - & - \\
\hline & 0.88 & 1.14 & - & - & 59.51 & 59.8 & - & - & 30.24 & 29.41 & - & - & 7.17 & 6.80 & - & - \\
\hline & 7.15 & 7.05 & - & - & 54.33 & 53.55 & - & - & 39.62 & 35.39 & - & - & 3.27 & 4.42 & - & - \\
\hline & 0.46 & 0.26 & - & - & 60.53 & 60.86 & - & - & 33.38 & 32.11 & - & - & 3.48 & 3.20 & - & - \\
\hline & 0.85 & 0.66 & - & - & 60.76 & 62.36 & - & - & 33.11 & 33.16 & - & - & 3.36 & 3.48 & - & - \\
\hline
\end{tabular}

Trans fatty acid (TFA), saturated fatty acid (SFA), monounsaturated fatty acids (MUFA), and polyunsaturated fatty acids (PUFA). 
Table 2 Supporting Information. GC-FID and ATR-FTIR data considering calibration (21 samples) and prediction (11 samples) steps of multivariate calibration for trans fatty acids isomers. Results are expressed as $\mathrm{g} / \mathbf{1 0 0} \mathrm{g}$ total fatty acids;

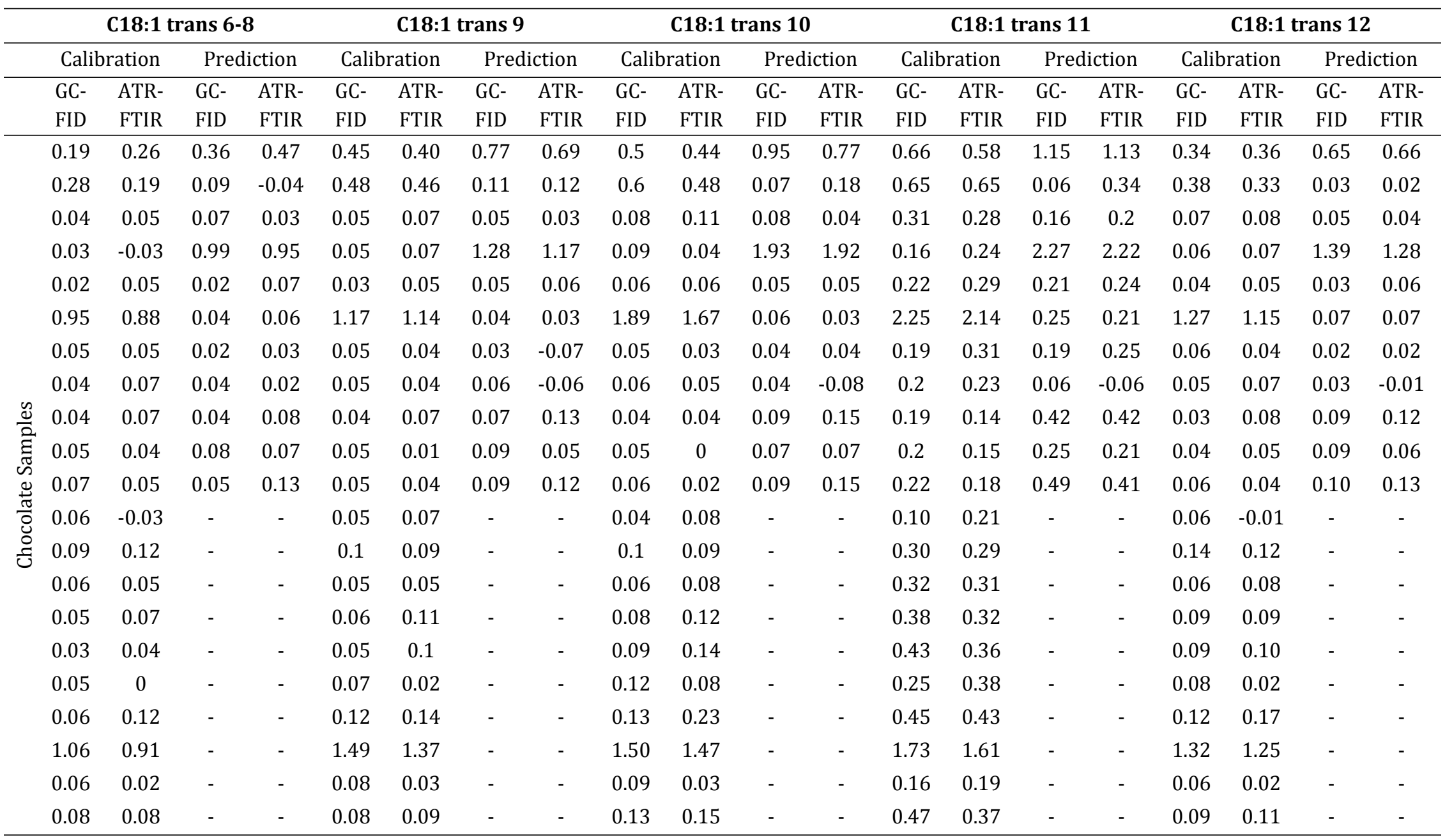


Figure 1 Supporting Information: Chromatograms of a chocolate sample containing high industrial trans fats contents and low milk contents (red) and a chocolate sample containing low industrial trans fats contents and high milk contents (black).

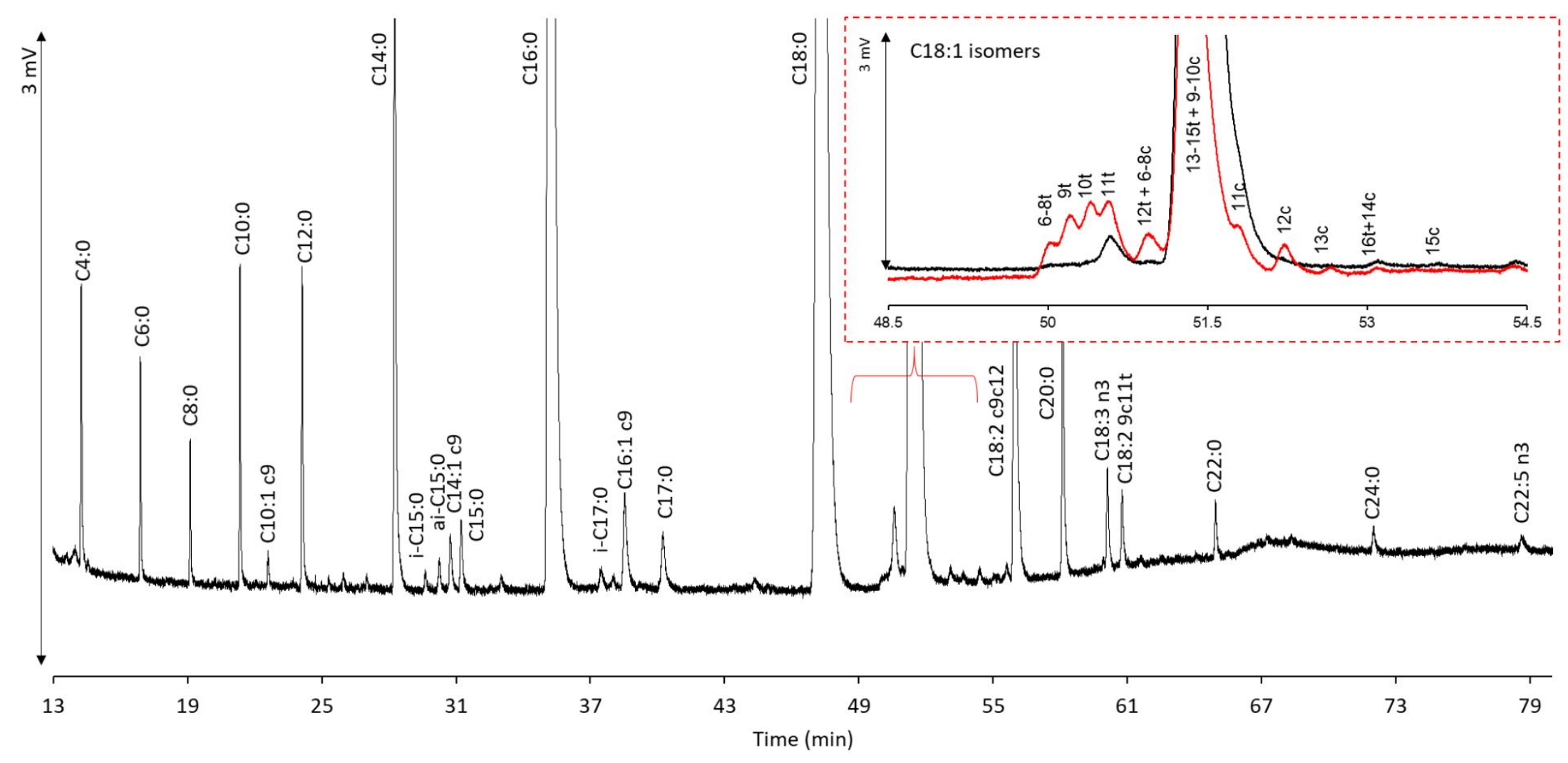

